\title{
Mindfulness-based waiting room intervention for osteopathic manipulation patients: a pilot randomized controlled trial
}

https://doi.org/10.1515/jom-2020-0186

Received July 17, 2020; accepted November 9, 2020;

published online February 24, 2021

\section{Abstract}

Context: Osteopathic manipulative treatment (OMT) and mindfulness-based interventions are both efficacious pain management strategies. Combining these two therapeutic approaches may offer added benefits to pain patients.

Objectives: To determine whether engaging in a mindfulness-based intervention before an OMT session improved OMT session outcomes.

Methods: Patients seeking OMT care from a single osteopathic physician at an integrative health clinic were recruited for this pilot randomized, controlled trial at an academic hospital. All patients scheduled for osteopathic structural evaluation and treatment with the provider from March 2019 to September 2019 were eligible and invited to participate during the reminder call before their visit. Participants were randomly assigned to listen to one of two audio recordings matched for length: (1) the history of osteopathic medicine, or (2) a guided mindfulness meditation practice. Patients completed surveys including numeric rating scales to measure mindfulness and embodied safety (a self-reported feeling that the patient's body was in a safe place) immediately before and after listening to the audio recording. A global pain rating report along with a sensation manikin (a digital human figure silhouette overlaid with a grid of 786 "sensation" pixels)

*Corresponding author: Adam Hanley, PhD, College of Social Work's Center, Mindfulness and Integrative Health Intervention Development, University of Utah, 395 South 1500 East \#111, Salt Lake City, UT 84112-8909, USA, E-mail: adam.hanley@utah.edu

Eric L. Garland, PhD, College of Social Work's Center, Mindfulness and Integrative Health Intervention Development, University of Utah, Salt Lake City, Utah, USA; and University of Utah, Salt Lake City Veterans Affair Medical Center, Salt Lake City, Utah, USA

Rebecca Wilson Zingg, DO, Division of Physical Medicine and Rehabilitation and the Huntsman Cancer Institute Wellness and Integrative Health Center, University of Utah, Salt Lake City, Utah, USA capturing both pleasant and unpleasant sensation were collected before and after the OMT session. Session satisfaction was also assessed with a single survey item.

Results: A total of 57 participants were enrolled in the study; however, 18 were unable to listen to the full audio recording and were excluded from further analysis. The final study sample consisted of 39 patients, with $19(48.7 \%)$ randomized to the history audio recording and 20 (51.3\%) randomized to the mindfulness recording. The mean age of patients was 57 years (standard deviation, 11.75 years); 25 (64.1\%) were women and 14 (35.9\%) were men. The most common primary pain location was the neck $(16 ; 41.0 \%)$, followed by back (12; 30.8\%) and joint (5; 12.8\%). Twenty (51.3\%) participants were cancer patients; 19 (48.8\%) did not have a cancer diagnosis. Practicing mindfulness before OMT increased patients' sense of mindful connection to ( $p=0.036)$ and safety within $(p=0.026)$ their bodies as well as their overall session satisfaction $(\mathrm{p}=0.037)$. Additionally, OMT paired with either study condition (mindfulness vs. history) decreased pain $(\mathrm{p}<0.001)$ and increased the ratio of pleasant to unpleasant sensations reported by patients $(\mathrm{p}<0.001)$. Finally, regardless of experimental condition (mindfulness vs. history), increased safety within the body predicted greater pain relief $(\beta=-0.33, p=0.035)$ and larger sensation ratio changes $(\beta=0.37, \mathrm{p}=0.030)$ at the OMT session's end. Additionally, increased mindful connection to the body predicted less pain $(\beta=-0.41$, $\mathrm{p}=0.005$ ) at the session's end.

Conclusions: This study demonstrated the feasibility of integrating a mindfulness-based intervention with OMT and results suggest that having patients listen to an audioguided mindfulness practice while waiting for their OMT session may increase their mindful connection to and safety within their bodies as well as their session satisfaction. This study also provides empirical evidence that OMT may increase the distribution of pleasant sensations reported by pain patients while decreasing the distribution of unpleasant sensations reported.

Keywords: chronic pain; meditation; mindfulness; OMT; osteopathic manipulative treatment; patient satisfaction. 
Between 20 and $30 \%$ of US adults have chronic pain [1, 2], and many seek pain relief through pharmacological and behavioral means. Opioid analgesics are among the primary pharmacological treatments of chronic pain; however, widespread opioid prescription has resulted in rapidly rising rates of opioid misuse, addiction, and overdose [3-5]. Consequently, clinical practice guidelines strongly suggest that behavioral pain management strategies should be firstline treatments for chronic pain [6-9]. In contrast with the poor long-term efficacy and iatrogenic risks of available analgesic pharmacotherapies [10], osteopathic manipulative treatment (OMT) [11-16] and mindfulness-based interventions are emerging as valuable methods of managing chronic pain [17-19]. The potential positive impact on cognitive impairment and psychiatric conditions (anxiety, depression) in patients with pain are additional areas of interest for applying these interventions.

Parallel lines of research have suggested that OMT and mindfulness-based interventions can be efficacious pain management strategies [20]. Limited empirical work has examined the efficacy of combining these two therapeutic approaches, despite theoretical work suggesting they are highly compatible [20]. Osteopathic philosophy espouses the fundamental tenets that the body is a unit, structure and function interrelate, and the body has self-healing and self-regulatory mechanisms [21]. In combination with lifestyle optimization (exercise, diet, sleep, and stress management), OMT uses a variety of techniques to address restrictions in the tissues and optimize how the body functions and clears disease [22]. In application to pain management, osteopathic manipulation aims to resolve dysfunction in the tissues that are contributing to pain generation. OMT may be considered in application to bony alignment, myofascial freedom and mobility, nerve transmission, ligamentous and membranous motion, visceral function, and fluid flow [23]. But, as pain is a biopsychosocial phenomenon [24, 25], management ideally addresses not only physical restrictions but also painassociated mental patterning in comprehensive manner, which includes but is not limited to emotional and memory-based associations, attentional restrictions that limit sensory perception, and physical sense of safety and embodiment (i.e., the patient's connection to his or her body). These phenomena may further contribute to and perpetuate pain as a global state of disease, dysfunction, and disintegration. As such, entering an OMT session in a state of mindfulness may enhance the therapeutic effects of OMT by broadening the scope of treatment to more directly include both body and mind.

Mindfulness is commonly defined as intentionally and nonreactively attending to the present moment [26]. The state of mindfulness can be cultivated through a variety of practices (e.g., mindfulness meditation, yoga, centering prayer), many of which use a "mindful object," such as the breath or the body, to stabilize attention in the present moment [27, 28]. Evidence suggests this attentional stance can provide stress [29, 30] and pain relief [29, 31-33]. By focusing on the breath or the body, pain is no longer the focus of attention and mental elaboration about pain (e.g., catastrophizing) decreases; thus, emotional reactions to pain also decrease [34-36]. Furthermore, by virtue of cultivating nonreactivity during mindfulness practice, pain and stress emerge as momentary and fleeting experiences passing through the larger field of awareness. As such, mindfulness may uniquely prepare patients for OMT by quieting the mind, relaxing the physical body, and loosening illness-related cognitive schemas that may help maintain pain in the body [20].

One study [37] of 147 patients empirically examined the effects of combining a mindfulness-based intervention with OMT; in it, core concepts from acceptance and commitment therapy (ACT) [38, 39], a third-wave cognitive behavioral therapy approach, were introduced to patients with pain during the first $15 \mathrm{~min}$ of six successive OMT sessions. Results indicated that the combined mindfulness-based intervention and OMT approach improved pain. Furthermore, patients found this combined approach acceptable, with 60 (95\%) of those completing the follow-up questionnaire reporting satisfaction with their overall experience and outcomes [37]. However, these results [34] were from was a single arm study without a randomized control condition, and thus, continued research is needed to investigate the additive benefit of a mindfulness-based intervention. Additionally, as ACT emphasizes a variety of therapeutic components including values clarification and activity planning $[38,39]$, it has thus far been unclear whether mindfulness provides unique additive benefit for OMT patients.

In this study, we sought to extend the valuable preliminary work of Carnes et al. [37] by examining whether engaging in a single, brief mindfulness meditation practice before an OMT session improved OMT session outcomes. We expected that patients randomized to listen to a brief audio-guided mindfulness meditation practice before their OMT session would evidence an increased state of mindfulness and embodied safety (a self-reported feeling that the patient's body was in a safe place) immediately after listening to the recording as well as greater pain relief and session satisfaction at the OMT session's end, compared with participants who listened to an audio-recording of the history of OMT. We also expected that increases in mindfulness and embodied safety occasioned by the presession 
mindfulness audio recording would predict greater pain relief and session satisfaction at the OMT session's end.

\section{Methods}

This study was a single site, two-arm, parallel group, pilot randomized controlled trial (trial registry: NCT04477278). All procedures were approved by the University of Utah institutional review board. Participants provided informed consent after reviewing an informed consent cover letter with study personnel.

Participants were patients seeking OMT from a single physician (R.W.Z.) at a National Cancer Institute designated comprehensive cancer center. The Wellness and Integrative Health Center, in which OMT was provided in this pilot study, exists on the first floor of the Huntsman Cancer Institute (HCI) where it is well-integrated with other outpatient clinics and inpatient treatment. While specializing in cancer care, this clinic provides certain services to select patients without cancer diagnoses (including caregivers and staff). Seamless integration within the $\mathrm{HCI}$ framework affords the benefits of broader awareness of past and current medical problems (through the electronic medical record), interprovider communication, and referral as needed for additional interventions and support. As potential concerns regarding implementation of mindfulness-based intervention included active psychosis or current uncontrolled substance abuse, all participating patients were screened for any changes in medical condition and other, current clinical symptoms upon intake; additional, more specific screening may be considered in future studies as indicated.

All patients scheduled for osteopathic structural evaluation and treatment with the provider from March 2019 to September 2019 were eligible and invited to participate during the reminder call before their visit. Both new and established patients were included and contributed to a study population that was heterogeneous regarding primary pain location, pain duration (acute vs. chronic), and pain medication use.

While waiting for their OMT session, participating patients were provided a tablet computer and a pair of noise-cancelling headphones. At the study's end, participants returned both the tablet and headphones to clinic staff. Computer-generated randomization within the Qualtrics survey platform (SAP SE) was used to allocate participants (1:1) to listen to one of two audio recordings matched for length (8 min and $13 \mathrm{~s}$ ): (1) the history of osteopathy, and (2) mindfulness meditation. The history of osteopathic medicine recording introduced patients to the founder, Andrew Taylor Still, and his philosophy of healing (Supplemental Material). The mindfulness meditation consisted of instruction in focused attention on breath and body sensations and metacognitive monitoring and acceptance of discursive thoughts, negative emotions, and pain. This script closely followed a standardized mindfulness induction script (Supplemental Material) validated in prior research on brief, in-person mindfulness-based interventions for pain in medical settings [40]. To minimize potential confounding factors (i.e., variance in vocal tone, pace, etc.) the same person (A.W.H.) was recorded reading both scripts. Before listening to the audio recording, patients completed a brief survey on the tablet via the Qualtrics survey platform that included a sensation manikin, which is described in a subsequent section of this article, and single items assessing relevant clinical symptoms (i.e., pain, fatigue, nausea, and health), mindfulness of the body, and embodied safety. Immediately after listening to the audio recording, but before their OMT session began, participants again completed the mindfulness of the body and embodied safety items. After their OMT session ended, participants again completed the sensation manikin and the pain numeric rating scale. Again, all surveys were completed on a tablet via the Qualtrics survey platform, which also securely stored all study data in an online repository. Deidentified participant data were analyzed using SPSS 26 and AMOS 26 (IBM). Study personnel and the osteopathic treatment provider (R.W.Z.) were blind to condition.

Osteopathic evaluation and treatment were performed by a single provider (R.W.Z.) who completed a predoctoral fellowship in osteopathic manipulative medicine during medical school training prior to a residency in Physical Medicine and Rehabilitation (PM\&R), and who is board certified through the American Board of Physical Medicine and Rehabilitation (ABPMR). Following residency completion, this provider (R.W.Z.) had continuous clinical practice at the Huntsman Cancer Institute Wellness and Integrative Health Center, where she provides osteopathic structural evaluation and management of musculoskeletal conditions as well as physician evaluations for an exercise-based program (POWER: Personal Optimism with Exercise Recovery) for cancer rehabilitation [41].

Patient OMT sessions lasted approximately 30-40 min. All pertinent regions of somatic dysfunction were noted in associated diagnoses; osteopathic findings that correlated with these regions were listed in addition to a more generalized, neuromusculoskeletal examination. Treatment was individualized based on these findings through implementation of a variety of osteopathic techniques (osteopathic cranial manipulative medicine, myofascial release, balanced ligamentous tension, balanced membranous tension, muscle energy, facilitated positional release, Still technique, counterstrain, and biodynamic osteopathy).

\section{Measures}

Sociodemographic and diagnostic information was obtained from patient medical records. Due to time limitations, only a single item was used for each of the mindfulness, embodied safety, and session satisfaction measurements. The item for each was selected for face validity.

Clinical symptoms: Self-reported pain, fatigue, nausea, and health were measured with individual respective items rated on a numeric rating scale (0-10), a widely used and validated approach to measuring clinical pain and related symptoms [40, 42].

Sensation manikin: The sensation manikin (Figure 1) is a digital human figure silhouette overlaid with a grid of 786 "sensation" pixels. Participants identified locations (i.e., grid pixels) on the manikin where they felt both pleasant and unpleasant sensations. Clicking once on any grid pixel turned that location blue, indicating a pleasant sensation. Clicking twice on any grid pixel turned that location red, indicating an unpleasant sensation. Each participant was instructed to color the entire area in which they were currently feeling sensation in their body. A clinically useful sensation ratio score was achieved by dividing the number of pleasant sensation pixels reported by the number of unpleasant sensation pixels reported [36]. 
Mindfulness of the body: Mindfulness of the body was measured with an individual item ("I felt in contact with my body") from the State Mindfulness Scale [43] rated on a numeric rating scale (0-10). Due to time limitations, only a single item was used from this scale and was selected for its face validity.

Embodied safety: Embodied safety was measured with an individual item ("I felt my body was a safe place") from the Multidimensional Assessment of Interoceptive Awareness [44] rated on a numeric rating scale $(0-10)$.

Session satisfaction: Session satisfaction was measured with an individual item ("I am satisfied with my treatment session") rated on a numeric rating scale (0-10).

\section{Statistical analysis}

Between-group differences in pain, sensation ratios, mindfulness of the body, and embodied safety were examined with a multivariate analysis of covariance (ANCOVA). Multivariate ANCOVA allows for the comparison of one or more grouping variables across multiple outcomes using a single statistical test, thereby decreasing family wise error $[45,46]$. Experimental condition (history of osteopathic medicine vs. mindfulness meditation) was used to predict the four outcomes after covarying baseline values and health status. In accordance with the classical ANCOVA approach for analyzing randomized controlled trial outcomes [47], covarying baseline values performs statistical matching on the prerandomization scores and ensures that comparisons of postrandomization values by treatment group are independent of baseline differences. Furthermore, given the heterogeneity in patients' diagnostic history and stage of care, we also adjusted for health status (i.e., cancer diagnosis and self-reported cancer symptom). While the hospital where this study was conducted specializes in cancer care, not all patients in this study had a cancer diagnosis. For those outcomes not differing by experimental condition, a repeated measures analysis of variance (ANOVA) was used to explore main effects of time. F-tests, which denote the ratio of two variances (i.e., variation between sample means/variation within the samples) [45], were used to evaluate the effect of experimental condition on pain, sensation ratios, mindfulness of the body, and embodied safety. The larger the F-statistic, the stronger the evidence that the experimental conditions differed. To facilitate interpretation of the F-statistics, we report standardized effect size estimates in the form of partial $\eta^{2}$ : small effect $=0.01$; medium effect $=0.06$; and large effect $=0.14$ [48]. Finally, path analysis was used to examine whether presession mindfulness-induced changes mediated the effect of condition on outcomes. Three fit indices were used to evaluate model fit: the $\chi^{2}$ value $\left(\chi^{2}\right)$, the comparative fit index (CFI) [49], and the root mean square error of approximation (RMSEA) [50]. A $\chi^{2}$ test with $p>0.05$, indicating consistency between the predicted model and observed data, along with CFI greater than 0.90 and RMSEA less than 0.08 , suggested adequate model fit.

\section{Results}

A total of 57 consecutive participants were enrolled in the study; however, 18 were unable to listen to their full audio recording and were excluded from further analysis. The final study sample consisted of 39 patients, with 19 (48.7\%) randomized to the history audio recording and 20 (51.3\%) randomized to the mindfulness recording. The mean age of patients was 57 years (standard deviation, 11.75 years); 25 (64.1\%) were women and 14 (35.9\%) were men. The most common primary pain location was the neck (16; 41.0\%), followed by back (12; 30.8\%) and joint $(5 ; 12.8 \%)$. Twenty (51.3\%) participants were cancer patients; 19 (48.8\%) did not have a cancer diagnosis. Most (32; 82.1\%) had previously received OMT from the study's osteopathic physician (R.W.Z.). Relatively mild pain levels were observed in this sample at baseline $(\bar{x}=4.49, \mathrm{SD}=1.79)$ [51] along with relatively high levels of self-reported mindfulness of the body $(\bar{x}=7.59, \mathrm{SD}=1.96)$. Demographic and baseline characteristics for all outcomes measures are shown in Table 1.

Across the entire sample at baseline, neither mindfulness of the body nor embodied safety were correlated with pain intensity or the sensation ratio. However, baseline sensation ratio scores and pain ratings were inversely correlated $(r=-0.46, \mathrm{p}=0.005)$. There were no between-group differences in any of the variables of interest at baseline (Table 1).

A multivariate ANCOVA was used to examine the effects of the presession audio-guided mindfulness practice on (1) patients' mindfulness of the body and embodied safety immediately after listening to the audio recording as well as (2) pain, sensation ratio (i.e., pleasant sensation/ unpleasant sensation), and session satisfaction at the session's end (Table 2).

With respect to the immediate effects of the presession audio recording, results revealed that experimental condition (mindfulness vs. history) had an effect on mindfulness of the body $\left(F_{1,31}=4.80, \mathrm{p}=0.036\right.$, partial $\left.\eta^{2}=0.134\right)$ and embodied safety $\left(F_{1,31}=5.48, \mathrm{p}=0.026\right.$, partial $\left.\eta^{2}=0.150\right)$, adjusting for baseline values and health status. Participants in the mindfulness condition reported greater mindfulness of the body (mindfulness sample mean $\bar{x}=8.05, \mathrm{SD}=2.19$; history $\bar{x}=7.11, \mathrm{SD}=2.51$ ) and embodied safety (mindfulness $\bar{x}=9.05, \mathrm{SD}=1.23$; history $\bar{x}=7.63$, $\mathrm{SD}=2.65$ ) compared with participants in the history condition.

With respect to the effects of the presession recording on session outcomes, results revealed that experimental condition (mindfulness vs. history) had an effect on session satisfaction $\left(F_{1,31}=4.76, \mathrm{p}=0.037\right.$, partial $\left.\eta^{2}=0.133\right)$, but not on pain $\left(F_{1,31}=0.33, \mathrm{p}=0.571\right.$, partial $\left.\eta^{2}=0.010\right)$ or sensation ratio $\left(F_{1,31}=0.13, \mathrm{p}=0.727\right.$, partial $\left.\eta^{2}=0.004\right)$, adjusting for baseline values and health. While participants in the mindfulness condition reported greater session satisfaction (mindfulness $\bar{x}=10.00, \mathrm{SD}=0.00$; history $\bar{x}=9.78, \mathrm{SD}=0.55$ ) 
Table 1: Baseline characteristics of participants by treatment group.

\begin{tabular}{|c|c|c|c|c|c|}
\hline & Total & History & Mindfulness & Test statistic & p-value \\
\hline $\mathrm{N}$ & 39 & 19 & 20 & & \\
\hline \multicolumn{6}{|l|}{ Sociodemographic characteristics } \\
\hline Age, years, mean (SD) & $57.42(11.75)$ & $57.89(8.39)$ & $56.95(14.60)$ & $t=0.25$ & 0.81 \\
\hline Age range, years & $25-76$ & $34-71$ & $25-76$ & $\ldots$ & \\
\hline Women, n (\%) & 25 (64.1\%) & $13(68.4 \%)$ & $12(60.0 \%)$ & $x^{2}=1.09$ & 0.58 \\
\hline Men, n (\%) & $13(33.3 \%)$ & $6(31.6 \%)$ & $7(35.0 \%)$ & & \\
\hline Primary pain location, n (\%) & & $\ldots$ & $\ldots$ & $x^{2}=1.38$ & 0.85 \\
\hline Back pain & $12(30.8 \%)$ & $6(31.6 \%)$ & $6(30.0 \%)$ & $\ldots$ & $\ldots$ \\
\hline Joint pain & 5 (12.8\%) & $3(15.8 \%)$ & $2(10.0 \%)$ & $\ldots$ & \\
\hline Neck pain & $16(41.0 \%)$ & $8(42.1 \%)$ & $8(40.0 \%)$ & $\ldots$ & $\ldots$ \\
\hline Other & $5(12.8 \%)$ & $2(10.5 \%)$ & $3(15.0 \%)$ & $\ldots$ & \\
\hline Cancer diagnosis, n (\%) & $20(51.3 \%)$ & $9(47.4 \%)$ & $11(55.0 \%)$ & $X^{2}=0.42$ & 0.52 \\
\hline Returning patient, n (\%) & $32(82.1 \%)$ & $16(84.2 \%)$ & $16(80.0 \%)$ & $x^{2}=0.98$ & 0.61 \\
\hline \multicolumn{6}{|l|}{ Baseline measures of study outcomes } \\
\hline Mindfulness of the body, mean (SD) & $7.59(1.96)$ & $7.79(1.87)$ & $7.40(2.06)$ & $t=0.62$ & 0.54 \\
\hline Embodied safety, mean (SD) & $7.54(2.44)$ & $7.26(2.85)$ & $7.80(2.02)$ & $t=0.68$ & 0.50 \\
\hline Pain, mean (SD) & $4.49(1.79)$ & $4.84(1.74)$ & $4.15(1.81)$ & $t=1.22$ & 0.23 \\
\hline Sensation ratio, mean (SD) & $0.95(2.00)$ & $1.06(1.90)$ & $0.84(2.11)$ & $t=0.34$ & 0.74 \\
\hline
\end{tabular}

SD, standard deviation.

compared with participants in the history condition, the clinical significance of this difference is unclear.

Follow-up repeated measures ANOVA revealed that although experimental condition did not impact pain or sensation ratio changes, main effects of time were observed for both outcomes (pain: $F_{1,36}=59.96, \mathrm{p}<0.001$, partial $\eta^{2}=0.625$; sensation ratio: $F_{1,37}=19.21, \mathrm{p}<0.001$, partial $\left.\eta^{2}=0.342\right)$. Regardless of experimental condition, OMT decreased pain (T1: $\bar{x}=4.50, \mathrm{SD}=1.81$; $\mathrm{T} 2: \bar{x}=2.71, S D=1.64$; $39.7 \%$ decrease) and increased sensation ratios (T1: $\bar{x}=0.95, \mathrm{SD}=1.99 ; \mathrm{T} 2: \bar{x}=10.36, \mathrm{SD}=13.68 ; 990.5 \%$ increase). The spatial distribution of unpleasant sensations (Figure 1) decreased from pre- to postsession (T1: $\bar{x}=29.21, \mathrm{SD}=41.51$; T2: $\bar{x}=6.82, \mathrm{SD}=10.49 ; 76.6 \%$ decrease) while pleasant sensations increased (T1: $\bar{x}=13.41, \mathrm{SD}=24.55 ; \mathrm{T} 2: \bar{x}=45.92$, $\mathrm{SD}=85.26 ; 242.4 \%$ increase).

Finally, path analysis (Figure 2) was used to examine relationships between the primary variables of interest.
The model fit the data well $\left(\chi^{2}=2.70, p=0.61, C F I=1.00\right.$, RMSEA $<0.001)$. Experimental condition had direct effects on change in mindfulness of the body, change in embodied safety, and session satisfaction. Additionally, change in mindfulness of the body and change in embodied safety had direct, inverse effects on change in pain. Furthermore, change in embodied safety had a direct positive effect on change in sensation ratio.

\section{Discussion}

The patient interview for pain management frequently focuses on pain itself. While much information is gathered regarding pain generation and exacerbating/relieving measures, this interrogation can also serve to further amplify the attentional focus and adhesiveness of mind to unpleasant sensation. According to Andrew Taylor Still,

Table 2: Study outcomes by treatment group.

\begin{tabular}{lrrrrr}
\hline & History & Mindfulness & Test statistic, $\boldsymbol{F}$ & p-Value & Effect size, partial $\eta^{2}$ \\
\hline Presession audio recording outcomes & & & & & \\
Mindfulness of the body, mean (SD) & $7.11(2.51)$ & $8.05(2.19)$ & 4.80 & 0.036 & 0.134 \\
Embodied safety, mean (SD) & $7.63(2.65)$ & $9.05(1.23)$ & 5.48 & 0.026 & 0.150 \\
Session outcomes & & & & & \\
Pain, mean (SD) & $2.94(1.80)$ & $2.50(1.50)$ & 0.33 & 0.571 & 0.010 \\
Sensation ratio, mean (SD) & $11.75(13.78)$ & $9.05(13.81)$ & 0.13 & 0.727 & 0.013 \\
Session satisfaction, mean (SD) & $9.78(0.55)$ & $10.00(0.00)$ & 4.76 & 0.037 & 0.133 \\
\hline
\end{tabular}

${ }^{\mathrm{a} P a r t i a l} \eta^{2}$ : small effect $=0.01$, medium effect $=0.06$, and large effect $=0.14$ [45]. SD, standard deviation. 


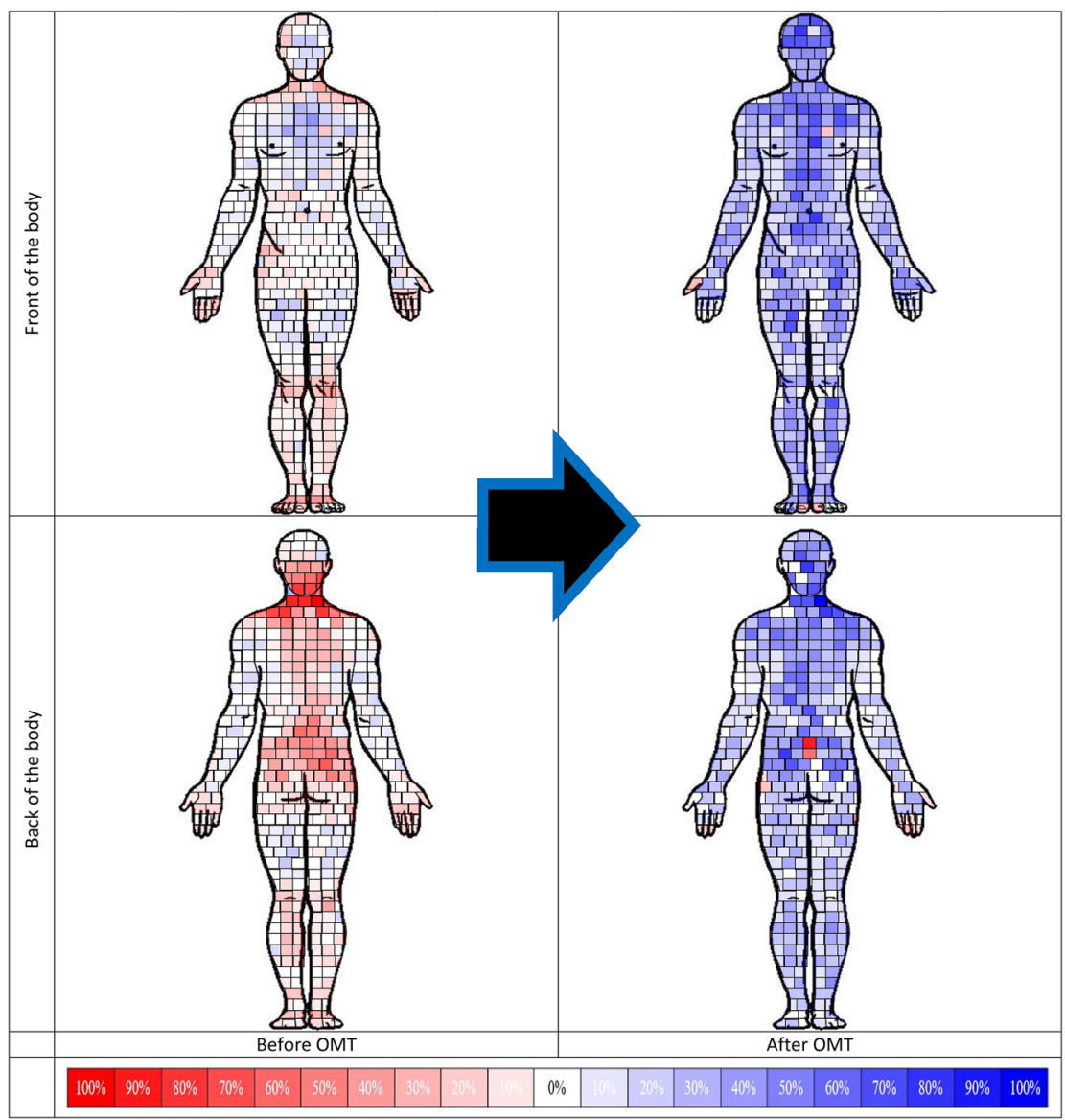

Figure 1: Sensation reports immediately before and after OMT. Blue represents pleasant sensations. Red represents unpleasant sensations. Color intensity reflects the frequency of sensation reports in a given sensation pixel. OMT, osteopathic manipulative treatment.
$\mathrm{MD}$, DO, the founder of osteopathic medicine: "To find health should be the object of the doctor. Anyone can find disease." [52] Through integration of OMT and mindfulness, focus is placed on reclaiming attentional control, broadening the physical map of pleasant and unpleasant sensation, resolving tissue restrictions to restore physiological integrity and function, and empowering the patient with tools that support health and wholeness in body and mind.

Results from the present study suggest that listening to a brief, audio-guided mindfulness instruction prior to OMT increased patients' sense of mindful connection to and safety within their bodies. These qualities of mindfulness are likely to facilitate healing, as physical traumas

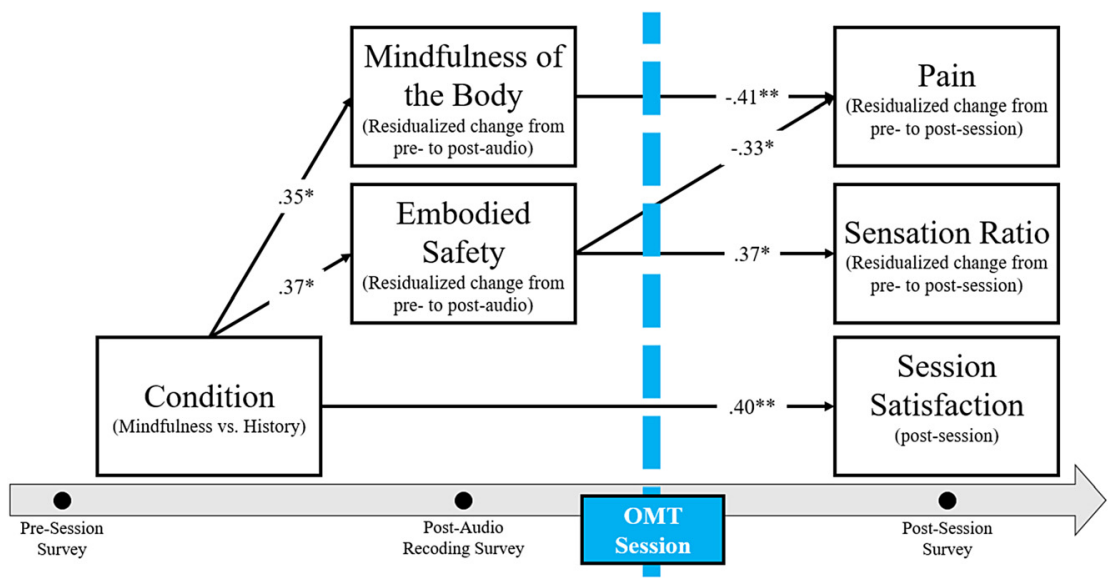

Figure 2: Path model depicting study timeline and relationships between primary variables of interest. Non-significant paths were removed from this figure to improve visual clarity. OMT; osteopathic manipulative treatment. 
(i.e., injury, disease), which are often the impetus for seeking OMT, invariably have both physical and psychological consequences. Indeed, injury and disease can make the body feel foreign and unsafe [53, 54], resulting in a tendency for patient to fear and avoid specific body parts or the body as a whole. As such, healing may be enhanced by including complementary, therapeutic interventions capable of cultivating a positive sense of connection with the body and nurturing the belief that the body is once again a safe place. Our results indicate that a brief, presession, audio-guided mindfulness practice can encourage positive psychological changes and more intensive mindfulness training could further amplify these outcomes. For example, future studies could increase the "dosage" of mindfulness practice by pairing a standardized 8-week mindfulness based intervention, such as Mindfulness Based Stress Reduction [26] or Mindfulness Oriented Recovery Enhancement [35], with regular visits. Additionally, regardless of experimental condition, increases in mindful connection to and safety within the body predicted greater pain relief and larger sensation ratio changes at the OMT session's end. OMT has demonstrated therapeutic value in pain management [11-14] and is a primary recommendation for common conditions such as low back pain [9], neck pain, and headaches (cervicogenic and migraine prophylaxis) [55]; however, an exclusive focus on the physical aspects of pain management and recovery neglects a vital dimension of healing. As a potentiating tool for embodiment and safety, mindfulness may serve as a cost-effective, inductive tool to encourage patient readiness for hands-on treatment and therapeutic release.

Through this pilot study, we explored how promoting a healing state of mind before OMT may have beneficial impact on patient experience. Indeed, beyond the acute, psychological benefits of the presession mindfulness practice, results from this study indicated that patients in the mindfulness group reported greater OMT session satisfaction, a clinical measure that merits ongoing evaluation in OMT of chronic pain conditions [12, 13, 15, 16]. However, path modeling revealed that neither mindfulness of the body nor embodied safety mediated the relationship between presession mindfulness and session satisfaction. Continued research is needed to investigate other salutogenic mechanisms of mindfulness-such as metacognitive awareness, nonreactivity to internal experiences, or disidentification with internal experiences [56]-that could explain why presession mindfulness resulted in greater postsession satisfaction.

Treatment satisfaction is closely associated with treatment adherence [57], and it may be that by coupling a mindfulness-based intervention with OMT, patients will be more likely to sustain their therapeutic involvement and realize more substantive and lasting therapeutic gains. This therapeutic involvement may include both passive, hands-on treatment as well as active engagement of change in home behaviors that support health and pain management (e.g., therapeutic exercises, dietary change, regular mindfulness practice, sleep optimization, and stress management).

Future research is needed to test this hypothesis, examining whether OMT patients who complete a presession mindfulness practice before their first OMT session continue hands-on treatment over time and report greater health benefits than those not receiving additional, mindfulness-based interventions. Through the combination of a mindfulness-based intervention with OMT, the patient is introduced to a self-care tool that may provide home-based, therapeutic use for ongoing pain management. Assessments in future research would ideally survey not only OMT follow-up but also the implementation of and adherence to home-based lifestyle and behavioral changes based on broader physician recommendations. These may include generalized conditioning and strengthening exercise; targeted, therapeutic exercise; dietary optimization; and ongoing mindfulness use. Such an assessment would inform the longer-term impact of mindfulness induction on motivation and behavioral change.

Some patients may dislike the presession mindfulness practice, prefer other types of presession psychological interventions, or dislike any type of presession intervention. In the current study, we chose to use an active control condition instead of a no-treatment control condition to account for nonspecific therapeutic factors, such as clinical time and attention, which may have influenced participant outcomes. Future studies are needed to directly compare whether adding a brief presession mindfulness practice to a standard OMT session outperforms a standard OMT session without any presession intervention. Furthermore, research is needed to examine individual characteristics that predict presession psychological intervention responsiveness and alternative presession psychological interventions (e.g., other styles of mindfulness practice, hypnotic suggestion, or breathing exercises) to better personalize presession psychological interventions for specific patients to maximize clinical outcomes.

OMT paired with either experimental condition decreased pain and increased sensation ratios (pleasant/ unpleasant). This finding exemplifies the potential therapeutic benefit from OMT in pain management. It also reveals the potential for OMT to foster attentional broadening within the body, loosening of the attentional anchor of 
pain, and experience of pleasant sensation. Through those mechanisms, treatment may eventually resolve the tension between disintegrated areas of the body (restriction and openness, pain and pleasantness) and restore a sense of wholeness to the patient. It has been well-documented that OMT can decrease pain $[11-14,56]$ and this study investigated whether OMT can change the spatial distribution of both unpleasant and pleasant sensations. Changes in the sensation ratio suggest that OMT fundamentally shifted patients' experiences of their bodies from disproportionately unpleasant before the OMT session $(\bar{x}=0.95)$ to disproportionally pleasant after the session $(\bar{x}=10.36)$ in this study. Sensation ratios below one indicate unpleasant sensations predominate in the body, while sensations ratios greater than one indicate pleasant sensations predominate. Visual inspection of the mean values and the sensation manikin (Figure 1) suggest that OMT had a powerful effect on patients' experience of embodied sensation; OMT increased the sensation ratio $990.5 \%$. Furthermore, it was not just decreases in unpleasant sensations driving this ratio change. While the spatial distribution of unpleasant sensations decreased by $76.6 \%$ from pre- to postsession, pleasant sensations increased by $242.4 \%$. These findings may suggest that strategies like OMT that are able to cultivate pleasant sensations may be valuable adjunctive treatments to more traditional pain management strategies that focus exclusively on pain reduction.

It is also worth highlighting the feasibility and efficacy of this intervention model. Patients randomized to the mindfulness experimental condition were able to increase their mindful connection to and safety within their bodies by listening to a brief, audio-recorded mindfulness practice while sitting in a waiting room in a busy medical hospital. There was no incentive for patients to participate in this study; patients were offered the opportunity to pass their time in the waiting room listening to an audio recording. As such, the implementation implications from this study are more direct than what would be expected from a study design that compensated patients for their participation. Additionally, with the majority of patients waiting more than $15 \mathrm{~min}$ for their appointments $[58,59]$, there was ample time to listen to a brief mindfulness recording, like the 8-min recording used in the current study. While studies $[58,60,61]$ in multiple medical settings indicate that longer clinic wait times translate into lower patient satisfaction, results from this study demonstrate that this waiting room time could be easily transformed into a space for contemplative practice that actually improves patient satisfaction-turning our waiting rooms into "breathing rooms" [62]. Furthermore, clinic flow was minimally disrupted as the audio recordings were loaded on the clinic's standard intake tablets. The only added burden was the purchase of two sets of headphones by the primary author (A.W.H.). Finally, no adverse experiences occurred during or after the presession mindfulness practice. In our clinical setting, integrating mindfulness-based intervention with the clinical visit appeared to be both effective and safe; however, additional safeguards such as in-person delivery of the mindfulness-based intervention, could be implemented with future studies to assure additional screening prior to starting the presession intervention Table 2.

\section{Limitations}

The main limitations in this study were the small sample size and the heterogeneity of the sample and treatment method. Future studies with larger sample sizes are needed to replicate the observed results. $G^{\star}$ Power 3.1 indicated that a sample of 54 patients would be needed to adequately power $(\beta=0.806)$ a replication study utilizing the same methodology, assuming an effect size of a similar magnitude to our smallest observed effect (partial $\eta^{2}=0.133$ ). Future studies are also needed to examine whether the observed results remain in more homogenous samples. Although we were able to control for factors likely to differ between OMT patients with and without a cancer diagnosis (i.e., fatigue, nausea, and health), future studies that evaluate a homogenous sample of individuals (cancerrelated pain, neck or back pain, acute or chronic pain) would be beneficial to ensure that these findings generalize to specific populations. While the provider (R.W.Z.) integrated various techniques to address individualized somatic dysfunction, this was not considered a weakness in study design, but rather an important application of OMT. Through this approach, treatment was tailored to the individual patient and facilitated by an osteopathic physician. Performance of an osteopathic structural examination and OMT by a single physician (R.W.Z.) through such individualized treatment may furthermore have served as a control. However, the limitations of this method are that treatment approach is not generalizable to a specific grouping of techniques or anatomical targets for a single pain condition.

Second, our study was limited by our use of single items from validated scales to assess constructs of interest (i.e., mindfulness of the body, embodied safety) instead of using the full scales from which these items were derived. While psychometric evidence supports the use of single- 
item scales to assess a range of psychological constructs [42, 63-65] including mindfulness [66, 67], neither the mindfulness of the body item nor the embodied safety item have been validated previously for use as single items. Thus, our mindfulness and embodied safety results should be interpreted with caution. The time-constraints imposed when researching in a busy academic cancer hospital limited our measurement approach. We abbreviated scales out of necessity for integration within the standard clinic flow. We chose the State Mindfulness Scale [43] and the Mindful Interoceptive Awareness Inventory [44] items included in this study for their face validity, selected by the lead author (A.W.H.) as the best available options for hypothesis testing. However, alternative items from these two scales may have yielded alternative results or functioned better than the chosen items. Future studies are encouraged to use the full scales should time allow. Future studies should also consider assessing clinical outcomes over a longer follow-up period, not just immediately after the OMT session.

Third, history of OMT experience and mindfulness practice involvement are individual difference variables that may have influenced the study's outcomes. Future researchers should gather more thorough demographic data. This sample's relatively high self-reported mindfulness at baseline may have influenced how willing participants were to notice body sensations and how well they could understand and follow the audio-recorded mindfulness practice. Examining whether similar results are observed in patient populations with lower baseline mindfulness scores is a valuable avenue for future research.

Fourth, emerging evidence suggests that brief, inperson mindfulness-based interventions for pain may be more efficacious than brief, audio-guided mindfulnessbased interventions [68]. Thus, future studies employing in-person mindfulness instruction before OMT may demonstrate more substantial effects, although the additional resource burden of in-person instruction should also be considered in study design selection.

\section{Conclusions}

Through this study, we demonstrated the feasibility of clinicbased integration of a mindfulness-based intervention with OMT. Benefits of listening to an audio-guided mindfulness practice before OMT may include an increased sense of embodiment and safety. Posttreatment benefit of a presession mindfulness-based intervention may include increased session satisfaction. However, because this was a pilot study, replication and future supportive research is needed to increase confidence in the present study's findings. Nevertheless, osteopathic providers may consider psychological interventions (mindfulness-based intervention, hypnotic suggestion, breathwork) as valuable, easily accessible, therapeutic tools to prepare patients for their treatment session and augment session satisfaction. This study also demonstrated the sizeable impact of OMT on pain and reduction in unpleasant sensation and augmentation of pleasant sensation and increase in overall sensation ratio (pleasant/unpleasant). This finding highlights the importance in pain management of incorporating interventions that augment pleasant sensation and support overall health and embodiment. With this, we turn from a singular focus on pain and disease back to a model that supports, facilitates, and augments the healthful capacity of the human being.

Research funding: Dr. Garland was supported by a grant from the National Institutes of Health (No. R01DA042033) during the preparation of this manuscript. The funders had no role in the design and conduct of the study; collection, management, analysis, and interpretation of the data; preparation, review, or approval of the manuscript; and decision to submit the manuscript for publication.

Author contributions: All authors provided substantial contributions to conception and design, acquisition of data, or analysis and interpretation of data; all authors drafted the article or revised it critically for important intellectual content; all authors gave final approval of the version of the article to be published; and all authors agree to be accountable for all aspects of the work in ensuring that questions related to the accuracy or integrity of any part of the work are appropriately investigated and resolved.

Competing interests: Dr. Garland is the Director of the Center on Mindfulness and Integrative Health Intervention Development. The Center provides Mindfulness-Oriented Recovery Enhancement (MORE), mindfulness-based therapy, and cognitive behavioral therapy in the context of research trials for no cost to research participants; however, Dr. Garland has received honoraria and payment for delivering seminars, lectures, and teaching engagements (related to training clinicians in MORE and mindfulness) sponsored by institutions of higher education, government agencies, academic teaching hospitals, and medical centers. Dr. Garland also receives royalties from National Association of Social Workers Press (Cary, NC) from the sale of books related to MORE. 
Informed consent: All participants in this study provided informed consent.

Ethical approval: This study was reviewed and approved by the Institutional Review Board at the University of Utah and was registered at clinicaltrials.gov (NCT04477278).

\section{References}

1. Dahlhamer J, Lucas J, Zelaya C, Nahin R, Mackey S, DeBar L, et al. Prevalence of chronic pain and high-impact chronic pain among adults - United States, 2016. MMWR Morb Mortal Wkly Rep 2018;67:1001-6.

2. Nahin RL. Estimates of pain prevalence and severity in adults: United States, 2012. J Pain 2015;16:769-80.

3. Guy JG, Zhang K, Bohm MK, Losby J, Lewis B, Young R, et al. Vital signs: changes in opioid prescribing in the United States, 2006-2015. MMWR Morb Mortal Wkly Rep 2017;66: 697-704.

4. Rudd RA. Increases in drug and opioid-involved overdose deaths-United States, 2010-2015. MMWR Morb Mortal Wkly Rep 2016;65:1445-52.

5. Schneiderhan J, Clauw D, Schwenk TL. Primary care of patients with chronic pain. J Am Med Assoc 2017;317:2367-8.

6. Department of Veterans Affairs. VA/DoD clinical practice guideline for opioid therapy for chronic pain; 2017. Available from: https://www.va.gov/HOMELESS/nchav/resources/docs/ mental-health/substance-abuse/VA_DoD-CLINICALPRACTICE-GUIDELINE-FOR-OPIOID-THERAPY-FOR-CHRONIC-PAIN508.pdf [Accessed 11 Dec 2020].

7. Brunkow A, Cannon M, Graff FS, Martin JL, Hausmann LR, McAndrew LM. Doctor recommendations are related to patient interest and use of behavioral treatment for chronic pain and addiction. J Pain 2020;21:979-87.

8. Dowell D, Haegerich TM, Chou R. CDC guideline for prescribing opioids for chronic pain-United States, 2016. J Am Med Assoc 2016;315:1624-45.

9. Qaseem A, Wilt TJ, McLean RM, Forciea MA. Noninvasive treatments for acute, subacute, and chronic low back pain: a clinical practice guideline from the American College of Physicians. Ann Intern Med 2017;166:514-30.

10. Chou R, Turner JA, Devine EB, Hansen RN, Sullivan SD, Blazina I, et al. The effectiveness and risks of long-term opioid therapy for chronic pain: a systematic review for a National Institutes of Health pathways to prevention workshop. Ann Intern Med 2015; 162:276.

11. Coronado RA, Gay CW, Bialosky JE, Carnaby GD, Bishop MD, George SZ. Changes in pain sensitivity following spinal manipulation: a systematic review and meta-analysis. J Electromyogr Kinesiol 2012;22:752-67.

12. Franke H, Franke J-D, Fryer G. Osteopathic manipulative treatment for nonspecific low back pain: a systematic review and metaanalysis. BMC Muscoskel Disord 2014;15:286.

13. Licciardone JC, Brimhall AK, King LN. Osteopathic manipulative treatment for low back pain: a systematic review and metaanalysis of randomized controlled trials. BMC Musculoskelet Disord 2005;6:43.
14. Rubinstein SM, De Zoete A, Van Middelkoop M, Assendelft WJ, De Boer MR, Van Tulder MW. Benefits and harms of spinal manipulative therapy for the treatment of chronic low back pain: systematic review and meta-analysis of randomised controlled trials. BMJ 2019;364:1689.

15. Licciardone JC, Schultz MJ, Amen B. Osteopathic manipulation in the management of chronic pain: current perspectives. J Pain Res 2020;13:1839.

16. Task Force on the Low Back Pain Clinical Practice Guidelines. American Osteopathic Association guidelines for osteopathic manipulative treatment (OMT) for patients with low back pain. J Am Osteopath Assoc 2016;116:536-49.

17. Cherkin DC, Sherman KJ, Balderson BH, Cook AJ, Anderson ML, Hawkes RJ, et al. Effect of mindfulness-based stress reduction vs cognitive behavioral therapy or usual care on back pain and functional limitations in adults with chronic low back pain: a randomized clinical trial. J Am Med Assoc 2016;315:1240-9.

18. Garland EL, Manusov EG, Froeliger B, Kelly A, Williams JM, Howard MO. Mindfulness-oriented recovery enhancement for chronic pain and prescription opioid misuse: results from an early-stage randomized controlled trial. J Consult Clin Psychol 2014;82:448.

19. Garland EL, Hanley AW, Riquino MR, Reese SE, Baker AK, Salas K, et al. Mindfulness-oriented recovery enhancement reduces opioid misuse risk via analgesic and positive psychological mechanisms: a randomized controlled trial. J Consult Clin Psychol 2019;87:927-40.

20. Mars TS, Abbey H. Mindfulness meditation practise as a healthcare intervention: a systematic review. Int J Osteopath Med 2010;13:56-66.

21. Dowling DJ, Martinke DJ. Chapter 3: the philosophy of osteopathic medicine. In: DiGiovanna EL, Schiowitz S, Dowling DJ, editors. An osteopathic approach to diagnosis and treatment, 3rd ed. Lippincott, Williams \& Wilkins; 2005:10-15 pp.

22. Seffinger MA, Hruby RJ, Rogers FJ, et al. Chapter 1: philosophy of osteopathic medicine. In: Foundations of osteopathic medicine: philosophy, science, clinical applications, and research, 4th ed. Wolters Kluwer Health Pharma Solutions (Europe) Ltd; 2018: 2-18 pp.

23. Gustowski S, Seals R, Gentry M. Osteopathic techniques: the learner's guide. New York, NY: Thieme; 2017.

24. Beneitez I, Nieto R. Do we understand pain from a biopsychosocial perspective? A review and discussion of the usefulness of some pain terms. Pain Manag 2017;7:41-8.

25. Garland EL. Pain processing in the human nervous system: a selective review of nociceptive and biobehavioral pathways. Prim Care 2012;39:561-71.

26. Kabat-Zinn J. Full catastrophe living. New York, NY: Delacorte Press; 1990.

27. Lutz A, Slagter HA, Dunne JD, Davidson RJ. Attention regulation and monitoring in meditation. Trends Cogn Sci 2008;12:163-9.

28. Vago DR, Silbersweig DA. Self-awareness, self-regulation, and self-transcendence (S-ART): a framework for understanding the neurobiological mechanisms of mindfulness. Front Hum Neurosci 2012;6:296.

29. Goyal M, Singh S, Sibinga EM, Gould NF, Rowland-Seymour A, Sharma R, et al. Meditation programs for psychological stress and well-being: a systematic review and meta-analysis. JAMA Intern Med 2014;174:357-68. 
30. Sedlmeier P, Eberth J, Schwarz M, et al. The psychological effects of meditation: a meta-analysis. Psychol Bull 2012;138: 1139.

31. Garland EL, Brintz CE, Hanley AW, et al. Mind-body therapies for opioid-treated pain: a systematic review and meta-analysis. JAMA Intern Med 2020;180:91-105.

32. Goldberg SB, Tucker RP, Greene PA, et al. Mindfulness-based interventions for psychiatric disorders: a systematic review and meta-analysis. Clin Psychol Rev 2018;59:52-60.

33. Hilton L, Hempel S, Ewing BA, et al. Mindfulness meditation for chronic pain: systematic review and meta-analysis. Ann Behav Med 2017;51:199-213.

34. Zeidan F, Vago DR. Mindfulness meditation-based pain relief: a mechanistic account. Ann N Y Acad Sci 2016;1373:114-27.

35. Garland EL. Mindfulness-oriented recovery enhancement for addiction, stress, and pain. Washington, D.C: NASW Press, National Association of Social Workers; 2013.

36. Hanley AW, Garland EL. Mapping the affective dimension of embodiment with the sensation manikin: validation among chronic pain patients and modification by Mindfulness-Oriented Recovery Enhancement. Psychosom Med 2019;81:612-21.

37. Carnes D, Mars T, Plunkett A, Nanke L, Abbey H. A mixed methods evaluation of a third wave cognitive behavioural therapy and osteopathic treatment programme for chronic pain in primary care (OsteoMAP). Int J Osteopath Med 2017;24:12-17.

38. Hayes SC, Strosahl KD, Wilson KG. Acceptance and commitment therapy: an experiential approach to behavior change. New York: Guilford Press; 1999.

39. Hayes SC, Luoma JB, Bond FW, Masuda A, Lillis J. Acceptance and commitment therapy: model, processes and outcomes. Behav Res Ther 2006;44:1-25.

40. Garland EL, Baker AK, Larsen P, et al. Randomized controlled trial of brief mindfulness training and hypnotic suggestion for acute pain relief in the hospital setting. J Gen Intern Med 2017;32: 1106-13.

41. Fitness and exercise / huntsman cancer Institute. Available from: https://healthcare.utah.edu/huntsmancancerinstitute/ wellness-support/wellness-integrative-health-center/fitnessprograms.php\#powerprogram [Accessed 23 Dec 2020].

42. Hawker GA, Mian S, Kendzerska T, French M. Measures of adult pain: visual analog scale for pain (vas pain), numeric rating scale for pain (nrs pain), mcgill pain questionnaire (mpq), short-form mcgill pain questionnaire (sf-mpq), chronic pain grade scale (cpgs), short form-36 bodily pain scale (sf-36 bps), and measure of intermittent and constant osteoarthritis pain (icoap). Arthritis Care Res 2011;63:S240-52.

43. Tanay G, Bernstein A. State Mindfulness Scale (SMS): development and initial validation. Psychol Assess 2013;25: 1286-99.

44. Mehling WE, Price C, Daubenmier JJ, Acree M, Bartmess E, Stewart A. The multidimensional assessment of interoceptive awareness (MAIA). PLoS One 2012;7:e48230.

45. Abu-Bader SH. Advanced and multivariate statistical methods for social science research with a complete SPSS guide. Oxford: Oxford University Press; 2016.

46. Keselman HJ, Huberty CJ, Lix LM, et al. Statistical practices of educational researchers: an analysis of their ANOVA, MANOVA, and ANCOVA analyses. Rev Educ Res 1998;68:350-86.
47. Frison L, Pocock SJ. Repeated measures in clinical trials: analysis using mean summary statistics and its implications for design. Stat Med 1992;11:1685-704.

48. Richardson JT. Eta squared and partial eta squared as measures of effect size in educational research. Educ Res Rev 2011;6: 135-47.

49. Bentler PM. Comparative fit indexes in structural models. Psychol Bull 1990;107:238-46.

50. Steiger JH. Structural model evaluation and modification: an interval estimation approach. Multivariate Behav Res 1990;25: 173-80.

51. Boonstra AM, Stewart RE, Köke AJA, et al. Cut-off points for mild, moderate, and severe pain on the Numeric Rating Scale for Pain in patients with chronic musculoskeletal pain: variability and influence of sex and catastrophizing. Front Psychol 2016;7. https://doi.org/10.3389/fpsyg.2016.01466.

52. Still AT. The philosophy and mechanical principles of osteopathy. Kansas City, Mo: Hudson-Kimberly Publishing Company; 1902.

53. Corbin JM. The body in health and illness. Qual Health Res 2003; 13:256-67.

54. Lindwall L, Bergbom I. The altered body after breast cancer surgery. Int J Qual Stud Health Well-Being 2009;4:280-7.

55. Tick H, Nielsen A, Pelletier KR, et al. Evidence-based nonpharmacologic strategies for comprehensive pain care: the Consortium Pain Task Force white paper. Explore 2018;14: 177-211.

56. Bernstein A, Hadash Y, Lichtash Y, Tanay G, Shepherd K, Fresco $D M$. Decentering and related constructs: a critical review and metacognitive processes model. Perspect Psychol Sci 2015;10: 599-617.

57. Barbosa CD, Balp MM, Kulich K, Germain N, Rofail D. A literature review to explore the link between treatment satisfaction and adherence, compliance, and persistence. Patient Prefer Adherence 2012;6:39-48.

58. Anderson RT, Camacho FT, Balkrishnan R. Willing to wait?: the influence of patient wait time on satisfaction with primary care. BMC Health Serv Res 2007;7:31.

59. McIntyre D, Chow CK. Waiting time as an indicator for health services under strain: a narrative review. Inquiry 2020; 57:1-15.

60. Bleustein C, Rothschild DB, Valen A, Valatis E, Schweitzer L, Jones R. Wait times, patient satisfaction scores, and the perception of care. Am J Manag Care 2014;20:393-400.

61. McMullen M, Netland PA. Wait time as a driver of overall patient satisfaction in an ophthalmology clinic. Clin Ophthalmol 2013;7: 1655-60.

62. Hanh TN. Peace is every step: the path of mindfulness in everyday life. New York: Random House; 2010.

63. Young Q-R, Nguyen M, Roth S, Broadberry A, Mackay MH. Singleitem measures for depression and anxiety: validation of the screening tool for psychological distress in an inpatient cardiology setting. Eur J Cardiovasc Nurs 2015;14:544-51.

64. Abdel-Khalek AM. Measuring happiness with a single-item scale. Soc Behav Pers 2006;34:139-50.

65. Robins RW, Hendin HM, Trzesniewski KH. Measuring global selfesteem: construct validation of a single-item measure and the Rosenberg Self-Esteem Scale. Pers Soc Psychol Bull 2001;27:151-61. 
66. Goldberg SB, Knoeppel C, Davidson RJ, Flook L. Does practice quality mediate the relationship between practice time and outcome in mindfulness-based stress reduction? J Couns Psychol 2020;67:115.

67. Donald JN, Atkins PW, Parker PD, Christie AM, Ryan RM. Daily stress and the benefits of mindfulness: examining the daily and longitudinal relations between present-moment awareness and stress responses. J Res Pers 2016;65:30-7.
68. McClintock AS, McCarrick SM, Garland EL, Zeidan F, Zgierska AE. Brief mindfulness-based interventions for acute and chronic pain: a systematic review. J Altern Complement Med 2019;25: 265-78.

Supplementary Material: The online version of this article offers supplementary material (https://doi.org/10.1515/jom-2020-0186). 